\title{
A Solution to the Binding Problem
}

\author{
Richard A. Mould \\ Department of Physics and Astronomy (Ret), State University of New York at Stony Brook, Stony Brook, \\ New York, USA \\ Email: richard.mould@stonybrook.edu
}

Received 4 February 2016; accepted 14 March 2016; published 17 March 2016

Copyright (C) 2016 by authors and Scientific Research Publishing Inc.

This work is licensed under the Creative Commons Attribution International License (CC BY). http://creativecommons.org/licenses/by/4.0/

cC) (i) Open Access

\begin{abstract}
Visual information about an object is widely distributed over the cortex. The problem of how this information gets reassembled in consciousness is the "binding problem". It is assumed in this paper that consciousness reads the distributed information as a laser reads a barcode; and that this solves the binding problem without resorting to oscillations, or synchronous signals, or any other form of mechanical association. Cortical distributions are made intelligible by consciousness that learns from childhood to recognize cortical arrays of single objects and project them onto the external world. An example shows how consciousness exercises its influence in the case of a wellknown line drawing. When an object is constructed by consciousness there is no guarantee that the resulting image will be anything like the original object of observation. However, there is reason to believe that most of the visual images of our surroundings reflect real properties of those surroundings. These images have a constancy about them that is not always conveyed by the sensory input, but consistency in the external world can be learned by consciousness that is able to override the incongruities of the senses.
\end{abstract}

\section{Keywords}

Binding Problem, Cerebral Cortex, Color Vision, Consciousness, Retina

\section{Introduction}

Pathways from the primary visual cortex to the central brain carry the bits and pieces of a visual object to various parts of the cerebral cortex. How these widespread parts get themselves associated into an object of perception is the classical "binding problem" of brain physiology. In the past, binding has been thought of as an engineering problem that correlates brain-wide information by using frequency and phase relationships, simultaneous occurrences, or other mechanical markers. That is, scattered pieces of an object that should be experienced together are the key to mechanisms that associate them together. No one knows how this supposedly happens, 
but theories abound [1]-[7]. This paper denies the validity of any mechanical solution. Instead, we rely on the ability of consciousness to directly recognize intelligible patterns on the cortex.

There are two parts to the classical binding problem: 1) mechanically connecting data belonging to the same object and 2) reading the result by consciousness. The first is unnecessary. We eliminate it by reading the original cortical distribution directly, rather than subjecting it to mechanical machinations that are then read by consciousness. Consciousness reads the entire cortical assembly as a laser reads a barcode, where it is the intensity at different locations that identifies a pattern. Focusing one's attention on an object intensifies the cortical features of the object - making it easy for consciousness to see a pattern and identify "something".

I assume that an individual is consciously aware of an object by the time its separate parts have reached the cerebral cortex. Newborns learn to read this information on the cortical surface. They learn over time to organize cortical events into a space-time array of separate objects, and to identify them. The resulting infantile construction is the framework for all subsequent (visual) learning about the outside world. The bars of an ordinary barcode are essential to the information obtained, and I say that the intensity of information on the cortex corresponds to the "bars" of the visual images formed by consciousness. The organizing principle is the intelligibility of consciousness.

This paper first describes color perception in terms of the stimulus to the retina and the cerebral cortex, showing how consciousness reads cortical intensities to experience a color-as opposed to mechanical binding accounts of color production. A well-known line drawing containing the gestalts of two women is then used to show why mechanical images of the women cannot be stored in the cortex, and that only the intelligibility of consciousness can read something into a drawing like this. And finally, it is argued that the constancy of objects in the external world of physics is best reproduced in mental images when conscious intelligibility is the unifier of data spread across the cortex.

\section{Color Perception}

There are three cones in the retina: red, green and blue. Neurological impulses representing red and green make it all the way to the cerebral cortex, but blue is attenuated because it degrades acuity; so only three impulses from the red and green cones plus the rods are available to create the full spectrum of colors. This is accomplished by combining the relative amplitudes of the three signals.

Figure 1 shows four relative amplitudes of the three impulses when the subject looks at four different colored panels - red, yellow, green, and blue. The vertical axis in each panel is the percentage of absorption of light by the three different kinds of receptors. Red cones (R) are represented by the dot on the left of the panel, green cones $(\mathrm{G})$ are represented by the dot on the right of the panel, and rods are represented by crosses $\times$ in the middle of the panel.

For the sake of argument, I am going to suppose that Figure 1 also represents the signature barcode of these four colors at the level of the cerebral cortex. It is unrealistic to represent the neurological complexities of the cerebral cortex with simple points and crosses, and there is an additional rich background that is not taken into account. Nonetheless, we will take advantage of the simplicity of Figure 1. The purpose of this paper is not compromised by letting the simple barcode in the retina stand-in for the more complex barcode in the cerebral cortex.

According to classical binding theory, the color yellow is formed in consciousness by first making a mechanical connection between the three locations in the yellow panel of Figure 1. The color is either known by a resonance in some frequency between the three locations in the figure, or by the simultaneity of the three locations in the figure, or by the use of more complex mechanisms that include memory and environmental background. For these schemes to work, the mechanism should also include the "intensity" of the signal at each location, because relative intensity is essential to the color; but these pre-conscious recipes don't generally do that. However, consciousness alone can read a cortical distribution like Figure 1, taking full account of intensities and their locations. It is like recognizing a constellation of stars in the night sky and reading it as an object of some sort.

\section{Gestalts}

Psychologists in the early part of the twentieth century noticed that same things can appear different to different observers or different to the same observer at different times. Figure 2 is a line drawing that illustrates the point. Looking at the drawing one way you see the portrait of a young woman who is looking over her left shoulder. 


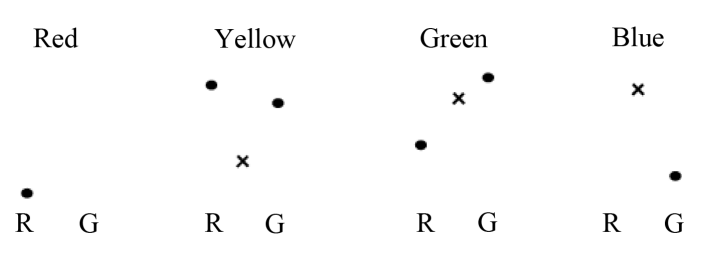

Figure 1. The barcode of color.

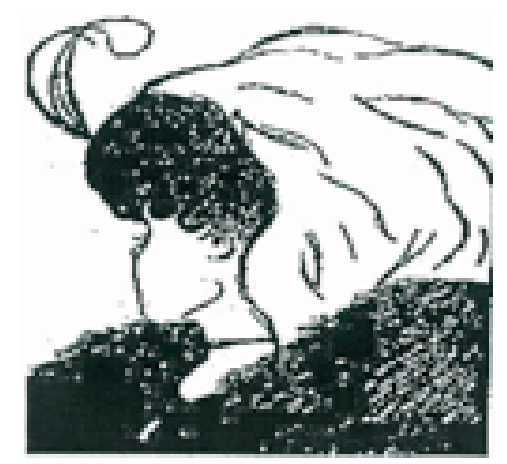

Figure 2. The Other Woman.

You can see her left cheek and her left ear, and she wears a necklace. Looking at the drawing in the other way, you see the left side of the face of an old woman with a large nose who is looking slightly downward. You can see her mouth and her chin that is partially buried in the fur of her coat. Each of these images is called a gestalt. A gestalt is an interpretation of given information, where alternative interpretations, or alternative gestalts, can be found in the same data.

From American Journal of Psychology 42.3 (July 1930) 444-445, copyright by the Board of Trustees of the University of Illinois, it is used with permission of the University of Illinois Press.

When someone looks at the Other Woman figure, the information in the drawing is spread over the observer's cerebral cortex in a way that does not include the image of the young woman or the old woman. The central brain does not produce a movie-like projection of the figure onto a neurological screen. Instead, incoming information is broken up in such a way as to destroy the images of the two women. I go a step further and say that the images do not even exist on the page. The reality of the page is an assembly of dark spots on a white background, and that is all. The image we see is our own projection - it is not on the page. If you believe the image is on the page, then think about which one is on the page and why you only see one at a time. This is a case in which the visual experience adds something of importance to the original featureless drawing. It adds one of the women. But neither woman is on the page or mechanically contained in the cortex. Both images are formed by consciousness that observes a pattern of bright constellations in the cortex.

It is argued in another place [8] that the images seen by consciousness might begin with complexity theory that allows diverse variables in a system to spontaneously generate distinct patterns [9]-[11]. That alone might allow preconscious creatures to organize gestalts to guide their behavior. But for conscious organisms, there is another requirement - intelligibility. It is of course possible that many of these patterns are made intelligible by virtue of the observer's past history alone, or by DNA alone.

However, in a novel situation a person's history and DNA do not apply. In these cases consciousness alone bestows intelligibility on a gestalt- - bringing it forward for the first time. Humans especially are prone to novel insights that alter their view of a subject or a situation, and in these cases only consciousness can endow the emerging gestalt with intelligibility; since there is no previous personal or ancestral experience that is relevant.

Notice that the choice between the two women in Figure 2 is discontinuous. One cannot go continuously from one image to the other. You snap into one image or into the other, so consciousness goes all the way with one interpretation or the other. The image of the young woman by itself is intelligible and the image of the old woman by itself is intelligible, but a transitional image between these two is not observed. If an image does exist between the two women arising from complexity theory, then consciousness rejects that image if it is not intelligible. That rejection is another way in which the intelligibility of consciousness exercises control. 


\section{Four-Dimensional Binding}

Time also plays a crucial role in binding. When an external stimulus sends information to the brain, different parts of that information reach different parts of the cerebral cortex at different times-because transit time varies along different neural pathways [12]. The times vary from $29 \mathrm{~ms}$ to $82 \mathrm{~ms}$, and consciousness must take in all of the data spread out over these times before completing the image. We designate $80 \mathrm{~ms}$ as the time consciousness waits for the slowest information to arrive at its destination [13]. This time delay applies across modalities as well as within modalities.

A bass drummer hears the sound of his drum at the same time he sees the stroke of his mallet. But someone far away may see the mallet stroke first and hear the sound later because light travels faster than sound. However, for someone who is reasonably close to the drummer, sight and sound are simultaneous as they are for the drummer. It turns out that there is no time delay if the difference in time between the visual and audio signals is less than $80 \mathrm{~ms}$. Within that time difference, the visual and audio signals are experienced simultaneously. Evidently consciousness waits up to $80 \mathrm{~ms}$ for the sound signal to be received before it forms a "conscious" impression of the drumming. Intelligibility in this case means: bringing all the data together (in time) that is "supposed to be together".

There is another example within the visual system alone. It is found that there is a time delay as a function of signal amplitude. High amplitude (i.e., bright) signals travel faster than low amplitude (i.e., dim) signals along neurons in the visual system [14]. This means that if a vertical stick moves horizontally with its ends illuminated more than its center, then the ends will appear to an observer to move ahead of the center of the stick-because the brain processes "bright" spots faster that "dim" spots. The stick should then appear to be bowed with its ends leading the way. However, it turns out that the stick appears to all observers as being straight up-and-down throughout its motion. Evidently the observer's consciousness waits for as much as $80 \mathrm{~ms}$ while it takes in all the information it needs to form an image of a vertical stick. Intelligibility in this case means: maintaining the "straightness of the stick". The slowest signal rattling around in the brain is given an opportunity by consciousness to catch up with the fastest signal, in order to preserve the integrity of the stick ${ }^{1}$.

As with the purely spatial binding problem, the 4D binding problem defies mechanical explanation. Eagleman describes two proposals that are intended to explain how the brain performs these temporal miracles of coordination; but as he points out, neither one of these schemes works [13]. Neither spatial nor temporal binding is achieved by mechanical manipulation. Instead I say that consciousness directly reads, or learns to read, the four-dimensional cerebral barcode by applying intelligence to the data.

\section{Mapping Reality}

More often than not, the intelligibility provided by consciousness gives a believable picture of external reality. The images we normally see around us have a "constancy" about them that is a creditable property of objects in the real world. It is believable that when a mallet hits a base drum it will immediately make a sound, and this immediacy is a property of the system - independent of the observer. Consciousness preserves this constancy for all orientations of the observer, for all weather and lighting conditions, for all velocities between the drum and the observer, and for any reasonable distance of the observer from the drum. Consciousness fails to provide constancy for all distances, but it does succeed out to a distance resulting in a time difference of about $80 \mathrm{~ms}$.

The straightness of a vertical stick in horizontal motion is also preserved by consciousness. There is constancy in the stick's shape that is independent of its velocity with respect to the observer and the lighting on the stick. This shape is also constant for different orientations of the stick, the time of day, and the distance between the stick and the observer. Consciousness preserves the constancy in the size, shape, and behavior of all the Newtonian objects in our environment, with dynamic exceptions such as the frequency of moving objects (color of light and pitch of sound); and this variation is documented by another kind of constancy-Doppler's law. All of physics is a matter of looking for constancy in equations of motion that are independent of observers. Consciousness therefore goes a long way toward preserving regularities in the world of physics; and to this extent, the images provided by consciousness are believable reproductions of the external world.

Consciousness smoothes over many glitches in the input data. Image constancy is preserved in spite of con-

${ }^{1}$ Consciousness waits until all the visual elements are present before forming an image, but this excludes a possible motor response that follows a completed image [15]. 
stant eye movements. It is preserved over the blind spot in the retina, and it is eventually preserved despite inverted lenses that turn the world up-side-down. These are all instances in which consciousness modifies sensory input to make a person's experience conform to its own version of the outside world. It usually puts things together the same way that nature puts them together. Contrary to this, we have also seen that consciousness sometimes fools us with optical illusions and it may add its own interpretation to reality by fabricating a gestalt that doesn't exist in the raw data.

\section{Differing Accounts}

It is claimed above that infants learn to recognize object configurations on the cerebral cortex, and that this establishes the blueprint for adult recognition. In another place the author gives a purely complexity-based account of this blueprint [8]. There is no conflict between these accounts. They may in fact complement one another.

\section{Conclusion}

The visual binding problem defies mechanical solutions. Visual data scattered over the cerebral cortex is said in this paper to be read by consciousness as a laser reads a barcode, and consciousness directly converts that reading into a visual image. The organizing principle of this conversion is intelligibility, a property that is intrinsic to consciousness and foreign to the purely mechanical system of the brain. It is shown how the reading of sensory data by consciousness can produce images that differ from the sensory input. They are generally more like external reality than the sensory input that would alone suggest, and sometimes they include the content that is not found in external reality.

\section{References}

[1] Crick, F.H.C. and Koch, C. (1990) Towards a Neurobiological Theory of Consciousness. Seminars in the Neuroscience, 2, 263-275.

[2] Dennett, D. (1991) Consciousness Explained. Little-Brown, Canada.

[3] Dennett, D. and Kinsbourne, M. (1992) Time and the Observer: The When and Where of Consciousness in the Brain. Behavior and Brain Sciences, 15, 183-201. http://dx.doi.org/10.1017/S0140525X00068229

[4] Engel, A.K., Fries, P., König, P., Brecht, M. and Singer, W. (1999) Temporal Binding, Binocular Rivalry, and Consciousness. Consciousness and Cognition, 8, 128-151.

[5] O'Regan, J.K. and Noë, A. (2001) A Sensorimotor Account of Vision and Visual Consciousness. Behavioral and Brain Sciences, 24, 939-1031. http://dx.doi.org/10.1017/S0140525X01000115

[6] O'Regan, J.K. (2011) Why Red Doesn't Sound Like a Bell? Oxford University Press, Oxford.

[7] Singer, W. (2001) Consciousness and the Binding Problem. Annals of the New York Academy of Sciences, 929, 123146. http://dx.doi.org/10.1111/j.1749-6632.2001.tb05712.x

[8] Mould, R.A. (2015) Cosmic Purpose and Human Consciousness. Resource Publications, Eugene.

[9] Bob, P. (2011) Brain, Mind, and Consciousness. Springer, New York. http://dx.doi.org/10.1007/978-1-4614-0436-1

[10] Heylighen, F. (2009) Complexity and Self-Organization. In: Bates, M.J. and Maack, M.N., Eds., Encyclopedia of Library and Information Sciences, Taylor and Frances, London, 1-17.

[11] Donderi, D.C. (2006) Visual Complexity: A Review. Psychological Bulletin, 132, 73-97. http://dx.doi.org/10.1037/0033-2909.132.1.73

[12] Schmolesky, M.T., Wang, Y., Hanes, B.D., Thompson, K.G., Leutgeb, S. and Schzll, J.D. (1998) Signal Timing across the Macaque Visual System. Journal of Neurophysiology, 79, 3272-3278.

[13] Eagleman, D.M. (2010) How Does the Timing of Natural Signals Map onto the Timing of Perception. In: Nijhawan, R. and Khurana, B., Eds., Space and Time in Perception and Action, Cambridge University Press, Cambridge, 216-231. http://dx.doi.org/10.1017/CBO9780511750540.014

[14] Maunsell, J.H., Ghose, G.M., et al. (1999) Visual Response Latencies of Magnocellular and Parvocellular LGN Neurons in Macaque Monkeys. Visual Neuroscience, 1, 144-149. http://dx.doi.org/10.1017/s0952523899156177

[15] Wasserman, G.S. (1985) Neural/Mental Chronometry and Chronotheology. Behavior and Brain Sciences, 8, 556-557. http://dx.doi.org/10.1017/S0140525X00045131 